\title{
Optical Gain and Radiative Current Density in Strain Compensated GaAsP/GaAsBi/GaAsP QWs Laser Structure
}

\author{
N Sfina ${ }^{1,3,}{ }^{*}$, A Jbeli, ${ }^{1,4}, J L$ Lazzari $^{2}$ and M Said ${ }^{1}$ \\ ${ }^{1}$ Laboratoire de la Matière Condensée et des Nanosciences (LMCN), Département de Physique, Faculté des Sciences de \\ Monastir, Université de Monastir, Tunisia \\ ${ }^{2}$ Centre Interdisciplinaire de Nanoscience de Marseille (CINaM), Aix-Marseille Université, France \\ ${ }^{3}$ Faculty of Science and art KKU Mahail Assir, King Khalid University, Saudi Arabia \\ ${ }^{4}$ College of Sciences \& Humanities of Ghat, Majmaah University, Saudi Arabia
}

\begin{abstract}
We have theoretically investigated the band structure engineering and optical gain spectra of dilute-bismide and bismide-free respectively in GaAsBi/GaAsP and GaAs/GaAsP laser structures. Subsequently, a type-I strain-compensated $\mathrm{GaAs}_{0.97} \mathrm{Bi}_{0.03} / \mathrm{GaAs}_{0.7} \mathrm{P}_{0.3}$ quantum well heterostructure which was optimized in terms of compositions and thicknesses has been studied by solving Schrodinger equation. We found that the incorporation of a small fraction of bismuth in the laser active region enhances significantly the optical properties such as the optical gain, which leads to a peak gain value of nearly $1000 \mathrm{~cm}^{-1}$ corresponding to a usual injection carrier concentration of $1.5 \times 10^{12} \mathrm{~cm}^{-2}$. A modal gain value equal to $80 \mathrm{~cm}^{-1}$ can be reached and radiative current density less than $50 \mathrm{~A} / \mathrm{cm}^{2}$ is expected. These latter results demonstrate that the dilute-bismide GaAsP/GaAsBi/GaAsP laser heterostructure arouses a great interest due to their high-power near-infrared light emitting diodes (LED) and laser diodes (LD) operating at room temperature (RT).
\end{abstract}

Keywords

Dilute-bismide, Band structure engineering, Quantum well laser, Near-infrared lasers, Optical gain

\section{Introduction}

In the last few years, the bismides III-V, in particular, GaAsBi has aroused a great attention in the aim of the realization of optoelectronic devices field such as infrared emitters, infrared detectors, laser diodes, solar cells and spintronics [1-6]. Indeed, the incorporation of few percents of $\mathrm{Bi}$ into conventional III-V semiconductors results in a remarkable lift of valence band, leading to a serious decrease of the band gap due to the valence band anti-crossing effects and thermal stability $[7,8]$. Thus, dilute bismide $\mathrm{Ga}(\mathrm{AsBi})$ based lasers diodes represent potential candidates for high efficiency infrared light sources.

Recently, $\mathrm{GaAs}_{1-\mathrm{x}} \mathrm{Bi}_{\mathrm{x}}$ materials grown on a gallium arsenide (GaAs) substrate have attracted intensive interests for variety of applications [9] such as Indium gallium arsenide nitride (InGaAsN) that is an intersting material for the active region of long wavelength edge-emitting lasers (LWEEL) and vertical cavity surface-emitting laser (VCSEL) since the high-quality InGaAsN structure could be grown on (GaAs) wafer [10]. It was shown both experimentally and theoretically that GaAs within an incorporated small amount of $\mathrm{Bi}$ induces a notable reduction of the band gap (60-88 $\mathrm{meV} / \mathrm{Bi} \%)$ and in particular an enhance of the spin-orbit splitting when the $\mathrm{Bi}$ concen- tration increases [11]. Dilute bismide GaAsBi based lasers diodes are promising candidates for high efficiency infrared (IR) light sources. Optically pumped laser diodes [12], Ga(AsBi) light-emitting diodes (LEDs) $[13,14]$ and electrically injected laser $\mathrm{Ga}(\mathrm{AsBi})(2.2 \% \mathrm{Bi})$ operation at $\mathrm{RT}$ with emission wavelength of $0.947 \mu \mathrm{m}$ have been demonstrated with promising optical material qualities [15].

Nevertheless, the incorporation of substantial fractions of bismuth (up to 11\%) into GaAs using Molecular Beam Epitaxy (MBE) has become possible using low growth temperatures

* Corresponding author: Noureddine Sfina, Laboratoire de la Matière Condensée et des Nanosciences (LMCN), Département de Physique, Faculté des Sciences de Monastir, Université de Monastir, Avenue de l'Environnement, 5019 Monastir, Tunisia; Faculty of science and art KKU Mahail Assir, King Khalid University, Saudi Arabia

Accepted: November 13, 2019

Published online: November 15, 2019

Citation: Sfina N, Jbeli A, Lazzari JL, et al. (2019) Optical Gain and Radiative Current Density in Strain Compensated GaAsP/ GaAsBi/GaAsP QWs Laser Structure. Recent Adv Photonics Opt 2(1):40-49 
and with a low As to Ga flux ratio $[16,17]$. On the other hand, the optical absorption and emission are essentially due to the existence of localized states below the continuum band edges of the $\mathrm{GaAs}_{1-\mathrm{x}} \mathrm{Bi}_{\mathrm{x}}$ alloys. Morover, the precedent effect could be due to the presence of disorder caused by spatial fluctuations of the band gap in the alloy composition [18], and to the thermal distortion caused by lattice vibrations [19]. The bismuth element is the heaviest atom of the same column as As whereas its size and core electronic structure are very different. Knowing all the preceding, it thus seems reasonable to presume that Bi could someway behave as an isoelectronic impurity in some III-V semiconductors and show unusual alloy properties. Whatever it has been foretold theoretically that the detached Bi impurity does not form a bound state in GaAs [20,21], it turned out that the presence of Bi clusters leads to the apparition of localized states within the band gap near the valence band in $\mathrm{GaAsBi}[18,22]$. In this context, the $\mathrm{GaAs}_{1-\mathrm{y}} \mathrm{P}_{\mathrm{y}} / \mathrm{GaAs}_{1-\mathrm{x}} \mathrm{Bi}_{\mathrm{x}} \mathrm{MQW}$ was designed and theoretically studied on an (001)-oriented GaAs substrate. We have investigated the optical properties of QW active regions consisting of compressively strained $\mathrm{GaAs}_{1-\mathrm{x}} \mathrm{Bi}_{\mathrm{x}}$ wells with tensile-strained $\mathrm{GaAs}_{1-\mathrm{y}} \mathrm{P}_{\mathrm{y}}$ barriers. A test structure consisting of $(15 \mathrm{~nm}) \mathrm{GaAs}_{0.7} \mathrm{P}_{0.3}^{1-y} /(7 \mathrm{~nm}) \mathrm{GaAs}_{0.97} \mathrm{Bi}_{0.03} /(15 \mathrm{~nm}) \mathrm{GaAs}_{0.7} \mathrm{P}_{0.3}$ was grown on a semi-insulating GaAs substrate under the same growth conditions as the active region of the laser structure. We previously reported growth studies which confirm the incorporation of $\mathrm{Bi}$ and $\mathrm{P}$ in the wells and barriers respectively [23]. The purpose of the present work is to exhibit the effects instigated by the bismide states, taking into account the influence of the modified band offset parameters (Table 1) $[24,25]$ on band structures and optical gain spectra for a strained GaAsP/GaAsBi/GaAsP QWs. This latter effect is supported by the fact that the modified offset parameters [26] play a fundamental role in energy dispersion, and thus in optical gain.

In this paper, we have calculated the characteristics and the properties of a dilute-bismide (dilute-Bi) laser structures based on strain-compensated GaAsP/GaAsBi/GaAsP multi-quantum wells on GaAs substrate embedded in AlGaAs designed to operate at around $0.96 \mu \mathrm{m}$ at RT. The theoretical performances of the dilute-Bi laser structure are estimated in terms of modal gain and radiative current density withdrawn from optical gain calculation in TE mode polarization emission. In addition, the impact of the incorporation of bismide in the active region of the dilute-Bi laser structure is evaluated and analyzed by the comparison to the results obtained on GaAsP/GaAs/GaAsP Bi-free laser structure suitable for $0.796 \mu \mathrm{m}$ emission at RT. Furthermore, The fundamental electron and holes states energy levels $\left(e_{1}, h h_{1}\right)$, the electron-hole wave-functions overlap, the maximum gain, the modal gain, the spontaneous emission rate Rsp and the radiative current density are calculated at RT versus the injected carrier density in order to test wether the features and efficiency of the dilute-Bi laser structure could make it operate at RT.

\section{Quantum Well Design Studies}

Our conceived laser structure is based upon of GaAsP/ GaAsBi/GaAsP strain-compensated type-I active region. The central $\mathrm{GaAs}_{0.97} \mathrm{Bi}_{0.03} \mathrm{QW}$ whom the thickness is $2.5 \mathrm{~nm}$ is laminated between two $10 \mathrm{~nm}$ thick $\mathrm{GaAs}_{0.7} \mathrm{P}_{0.3}$ QWs which are in turn sandwiched by two $20 \mathrm{~nm}$ thick GaAs barriers that are lattice matched to the GaAs substrate. This structure is analyzed by comparing results to those obtained on Bi-free GaAsP/GaAs/GaAsP emitting at the same wavelength 0.796 $\mu \mathrm{m}$ at RT. The Figure 1 illustrates the schematic band diagram within the fundamental symmetric electron $e_{1}$ and hole $h h_{1}$ energy levels and their respective wave functions for the two laser structures, (a) Dilute-Bi and (b) Bi-free. The energy levels of these subbands have been calculated by solving the one-dimensional Schrödinger equation assuming the envelope function approximation $[27,28]$. The physical parameters used in the calculations are listed in Table 1 . The conduction and valence band discontinuities $\Delta E_{c}$ and $\Delta E_{v}$ at hetero-interfaces, which are key parameters for the energy band diagram construction, were calculated by the density functional theory (DFT) and their values are listed in Table $2[29,30]$. Also, the band gaps of $\mathrm{GaAs}_{1-\mathrm{y}} \mathrm{P}_{\mathrm{y}}$ and $\mathrm{GaAs}_{1-\mathrm{x}} \mathrm{Bi}_{x}$ strained to GaAs (001) were calculated using the following relationships, according to the DFT calculations and experimental fits are quoted from references [31,32].

Table 1: Material parameters used in the calculations at $300 \mathrm{~K}$. They are taken from References $[24,25]$.

\begin{tabular}{|l|l|l|l|l|l|l|}
\hline Parameters (T = $\mathbf{3 0 0} \mathbf{~ k})$ & Symbols & GaAs & GaP & AlAs & AlP & GaBi \\
\hline Lattice constant & $\alpha(\AA)$ & 5.653 & 5.4505 & 5.6622 & 5.4672 & 96.324 \\
\hline Band Gap & $E_{g}(\mathrm{eV})$ & 1.42 & 2.994 & 3.099 & 3.63 & -1.45 \\
\hline Electron mass & $m^{*} / m_{0}$ & 0.0665 & 0.13 & 0.15 & 0.22 & 0.039 \\
\hline Luttinger parameters & $V_{1}$ & 6.85 & 4.05 & 3.4 & 3.35 & 13.4 \\
\hline & $V_{2}$ & 2.1 & 0.49 & 0.82 & 0.71 & 4.7 \\
\hline Deformation potentials & $a_{v}(\mathrm{eV})$ & 1.16 & -8.2 & 2.47 & 25.7 & -6.85 \\
\hline & $a_{c}(\mathrm{eV})$ & -7.17 & -1.7 & -5.64 & 23.0 & 0.79 \\
\hline Elastic constant & $C_{11}(\mathrm{GPa})$ & 1221 & 1405 & 1250 & 1330 & 119.2 \\
\hline & $C_{12}(\mathrm{GPa})$ & 566 & 620.3 & 534 & 630 & 59.9 \\
\hline Spin-orbit energy & $\Delta_{0}(\mathrm{eV})$ & 0.341 & 0.08 & 0.28 & 0.07 & 2.15 \\
\hline Refractive indices & $n_{o p}$ & 3.3 & 3.363 & 3.677 & 2.348 & 4.532 \\
\hline
\end{tabular}



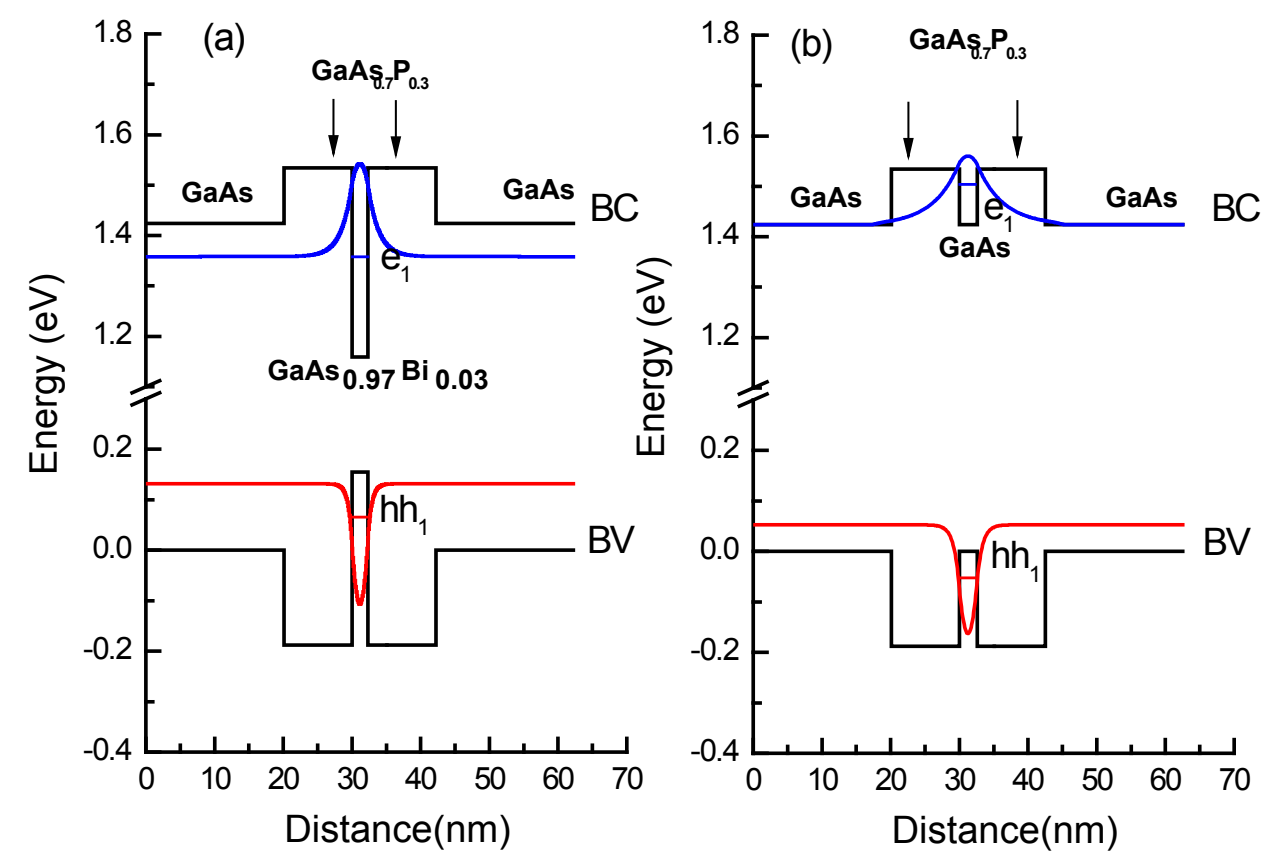

Figure 1: Band profiles, energy levels and selected wave function components for one period of the laser structure; (a) (20 nm) GaAs/ $(10 \mathrm{~nm}) \mathrm{GaAsP} /(2.5 \mathrm{~nm}) \mathrm{GaAsBi}$ designed for $0.96 \mu \mathrm{m}$ and $(\mathrm{b})(20 \mathrm{~nm}) \mathrm{GaAs} /(10 \mathrm{~nm}) \mathrm{GaAsP} /(2.5 \mathrm{~nm})$ GaAs designed for $0.796 \mu \mathrm{m}$.
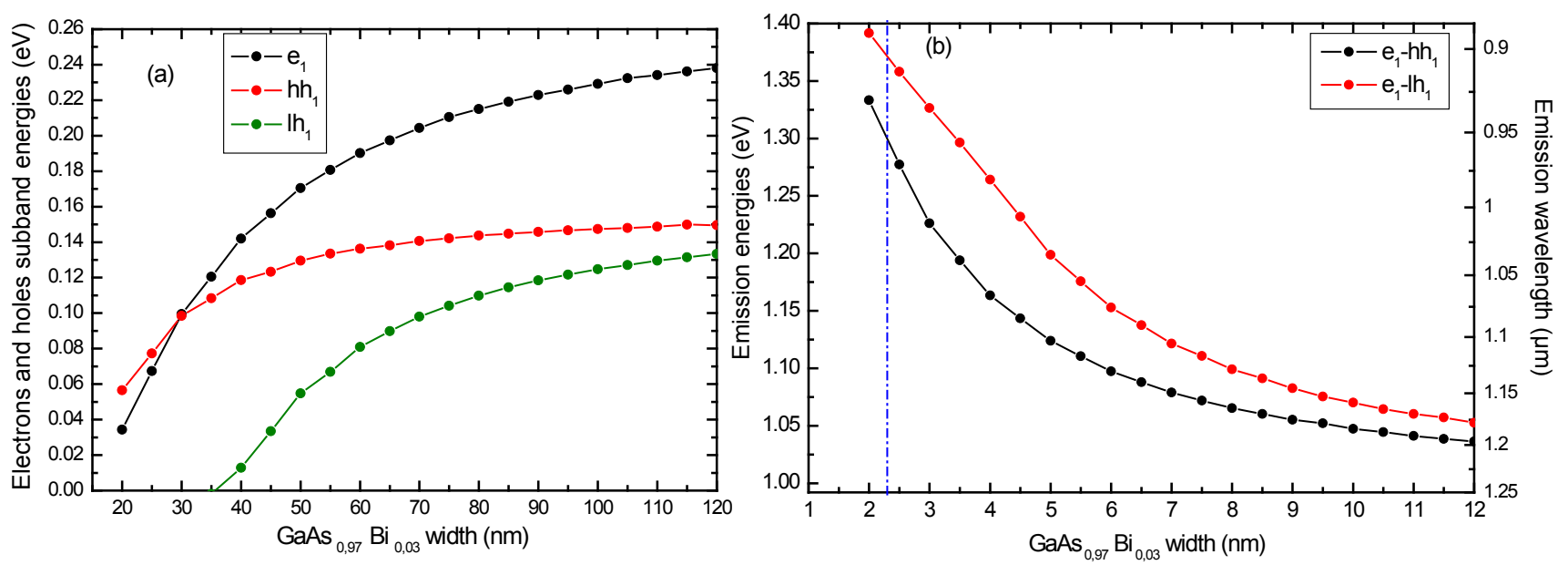

Figure 2: (a) Electron, heavy hole and light hole confinement-energies for the GaAsP/GaAsBi/GaAsP quantum well on relaxed GaAs as a function of the $\mathrm{GaAs}_{0.97} \mathrm{Bi}_{0.03}$ well width; (b) Emission energies $\mathrm{e}_{1}-\mathrm{hh}_{1}$ and $\mathrm{e}_{1}-\mathrm{lh}_{1}$ versus the $\mathrm{GaAs}_{0.97} \mathrm{Bi}_{0.03}$ quantum well width at $300 \mathrm{k}$.

Table 2: Band offset values of different hetero-interfaces of the valence $\Delta E_{v}(\mathrm{meV})$ and the conduction $\Delta E_{c}(\mathrm{meV})$ bands, respectively extracted from references [29].

\begin{tabular}{|l|l|l|l|}
\hline Interfaces & $(\boldsymbol{y}, \mathbf{z})$ & $\Delta E_{c}(\mathbf{m e V})$ & $\Delta E_{v}(\mathbf{m e V})$ \\
\hline \multirow{2}{*}{$\mathrm{GaAs} / \mathrm{GaAs}_{1-\mathrm{z}} \mathrm{Bi}_{z}$} & $(0,0.03)$ & -265 & 155 \\
\cline { 2 - 4 } & $(0,0.042)$ & -72 & 231 \\
\hline \multirow{2}{*}{$\mathrm{GaAs}_{1-\mathrm{y}} \mathrm{P}_{\gamma} / \mathrm{GaAs}_{1-\mathrm{z}} \mathrm{Bi}_{z}$} & $(0.297,0.016)$ & -231 & -96 \\
\cline { 2 - 4 } & $(0.3,0.03)$ & -375 & -188 \\
\hline & $(0.292,0.042)$ & -280 & -198 \\
\hline
\end{tabular}

$E_{g}\left(\operatorname{GaAs}_{1-y} P_{y}\right)=1.42+0.811 y-0.118 y^{2}-0.059 y^{3}$

$$
E_{g}\left(G a A s_{1-x} B i_{x}\right)=1.42-6.429 x+9.293 x^{2}+14.224 x^{3}
$$

The dilute- bismide compound induces deep GaAsP/ GaAsBi/GaAsP QW with high band offset value leading to good confinement of both types of carriers. The conduction and valence band-offse between GaAs and GaAs$\mathrm{Bi}$ have been calculated and are, respectively, $\Delta E_{c}=-265$ $\mathrm{meV}$ and $\Delta E_{c}=155 \mathrm{meV}$. besides, in both the dilute-Bi or $\mathrm{Bi}$-free laser structure, the active region is composed of type-I GaAsP/GaAsBi/GaAsP QWs. Subsequently, in this non-aligned configuration, the effective band gap seems to be independent of temperature and the laser emission wavelength could be easily tuned by tweaking the layer thickness of the GaAsP/GaAsBi/GaAsP period. These the- 
oretical results indicate that a correctly selected $\mathrm{GaAs}_{1-\mathrm{y}} \mathrm{P}_{\mathrm{y}}$ barrier provide band offsets much better than the thermal energy of RT even for the $\mathrm{GaAs}_{1-\mathrm{z}} \mathrm{Bi}_{2}$ system with minor $\mathrm{Bi}$ incorporation. Furthermore, it is important to note that the potential barrier introduced by $\mathrm{GaAs}_{1-\mathrm{y}} \mathrm{P}_{\mathrm{y}}$ could prevent carrier leakage, which will in opportunity, be expected to lead to an enhanced carrier injection efficiency and a better threshold current density at elevated temperature similar to that reported by reference [33] for InGaAsN lasers.

The energy levels of the different subband edges are represented in Figure 2. The latter plot shows that the quantum confinement energies of both types of carriers collapse with reduction of the $\mathrm{GaAs}_{0.97} \mathrm{Bi}_{0.03}$ layer width and attempt to stabilize from a thickness of $7 \mathrm{~nm}$. Besides, it is shown on Figure $2 a$ that for the $\mathrm{GaAs}_{0.97} \mathrm{Bi}_{0.03}$ layer thickness values less than the critical value of $7 \mathrm{~nm}$, the energies vary in a nearly linear manner and rise with the $\mathrm{GaAs}_{0}$. ${ }_{97} \mathrm{Bi}_{0.03}$ layer width. In order to justify this behavior, we stipulate that the wave function of this state is localized in the $\mathrm{GaAs}_{0.97} \mathrm{Bi}_{0.03}$ layer mostly. It can also be seen on Figure $2 \mathrm{~b}$, that the emission energies decrease until $\mathrm{GaAs}_{0.97} \mathrm{Bi}_{0.03}$ layer thickness of $3.8 \mathrm{~nm}$ and then become constant and the interband transitions correspond then to the wavelength of $0.96 \mu \mathrm{m}$. Now that the $\mathrm{GaAs}_{0.97} \mathrm{Bi}_{0.03}$ layer is a deep quantum well for the holes, the wave function of the these carriers ground state is situated also in the $\mathrm{GaAs}_{0.97} \mathrm{Bi}_{0.03}$ layer. Thus, the transitions in such heterostructures are direct in the coordinate space (see Figure 1).

The present laser structure designed with bismide for infrared emission is made of $\mathrm{GaAsP} / \mathrm{GaAsBi} / \mathrm{GaAsP}$ type-I quantum wells. A $22 \AA$ thick GaAsBi layer sandwiched by two $20 \AA$ thick $\mathrm{GaAs}_{0.7} \mathrm{P}_{0.3}$ layers. Each of $\mathrm{GaAsP} / \mathrm{GaAsBi} / \mathrm{GaAsP}$ quantum wells is separated by a $200 \AA$-thick GaAs spacer layer lattice-matched to the substrate. This feature confirms the bi-dimensional behavior of carriers in each MQW period. The dilute-bismide compound induces deep wells with high conduction and valence band offset values allowing strong confinement of both types of carriers. The GaAsP and GaAsBi layer thicknesses are chosen to exhibit laser emission around $0.96 \mu \mathrm{m}$ at RT with the highest electron-hole wave-function overlap for the GaAsP/GaAsBi/GaAsP. This latter structure's active region is embedded in an undoped $100 \AA$-thick $\mathrm{Al}_{0}$. ${ }_{18} \mathrm{Ga}_{0.82}$. As waveguide layer whose the geometry enhances the optical confinement factor while minimizing free-carrier absorption losses in the $\mathrm{Al}_{0.3} \mathrm{Ga}_{0.7} \mathrm{As}$ cladding layer. This layer provides an improve of the optical confinement and high type-II band-offsets and let hope a reduction of the carriers leakage current. The Figure 3 shows the schematic band diagram of the dilute bismide laser structure with the fundamental electron $\mathrm{e}_{1}$ and heavy holes $\mathrm{hh}_{1}$ energy levels and their respective wave functions. The computation was performed using the Schrödinger equation and assuming the envelope function approximation [27].

\section{Optical Gain and Radiative Current Calculation}

The optical characteristics of the dilute-Bi GaAsP/GaAsBi/ GaAsP structure are optimized in terms of modal gain and the threshold current density which is returned from optical gain calculation in TE mode polarization emission. For a quantum well, when $k \rho=0$, the optical gain energy spectra can have the following expression [34].

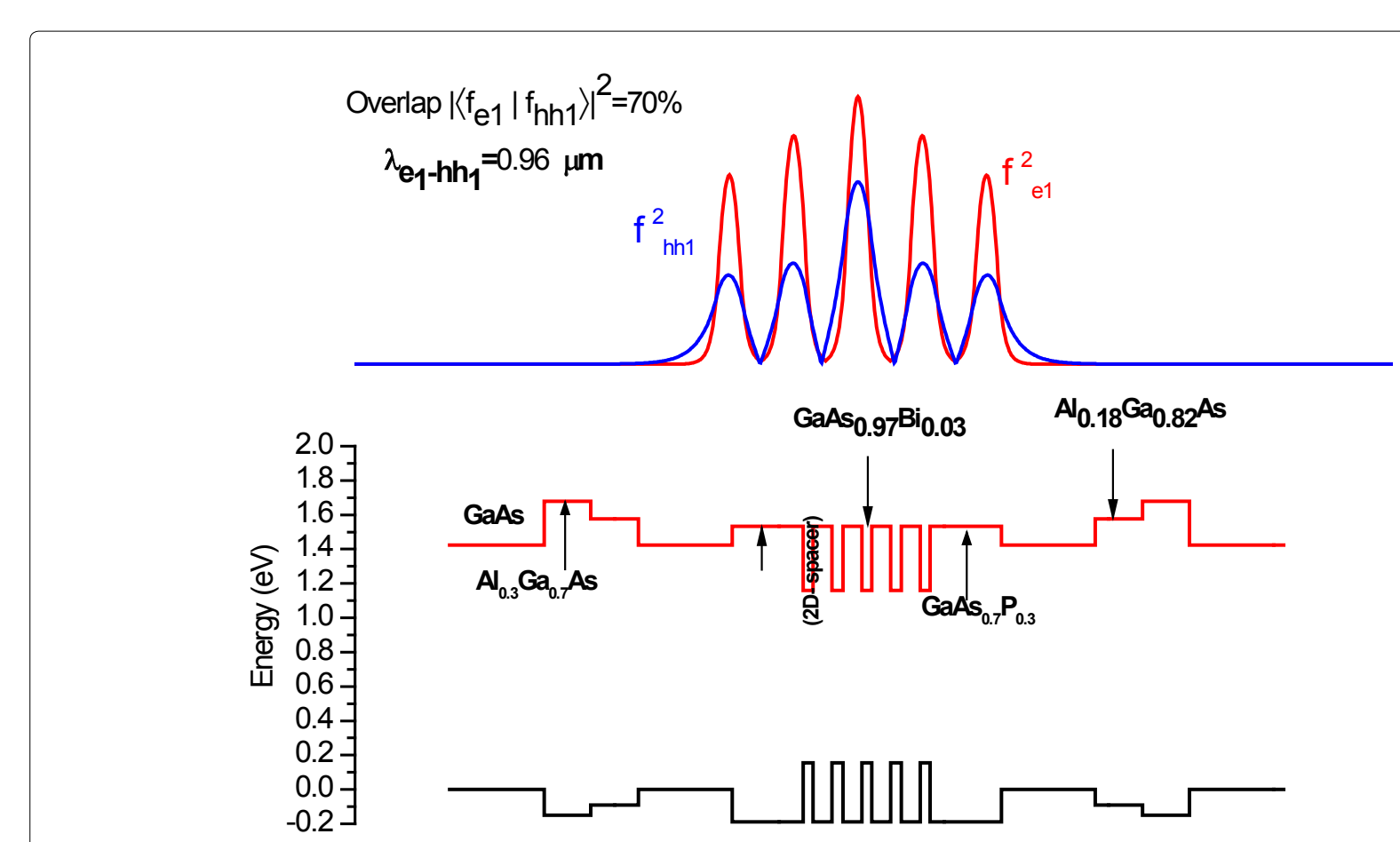

Figure 3: Conduction and valence band profiles of the laser structure built-on $\mathrm{Al}_{0.3} \mathrm{Ga}_{0.7} \mathrm{As}(200 \AA) / \mathrm{Al}_{0.18} \mathrm{Ga}_{0.82} \mathrm{As}(100 \AA) / \mathrm{GaAs}(200 \AA) /$ $\left.\operatorname{GaAs}_{0.7} \mathrm{P}_{0.3}(150 \AA) / \mathrm{GaAs}_{0.7} \mathrm{P}_{0.3}(20 \AA)\right) / \mathrm{GaAs}_{0.97} \mathrm{Bi}_{0.03}(22 \AA) / \mathrm{GaAs}_{0.7} \mathrm{P}_{0.3}(20 \AA)$ MQWs on GaAs substrate. The fundamental electron $\left(\mathrm{e}_{1}\right)$ and heavy-hole $\left(\mathrm{hh}_{1}\right)$ presence probability densities are displayed on the lower part. The fundamental $\mathrm{e}_{1}$-hh $_{1}$ optical transition is expected at $0.96 \mu \mathrm{m}$ at RT (300 k) with a wave function overlap value $\left|\left\langle f_{e 1} \mid f_{h h 1}\right\rangle\right|^{2}=70 \%$. 


$$
G(\hbar \omega)=G_{0} \sum_{i j}\left|I_{i j}\right|^{2}\left[f_{c i}(\hbar \omega)-f_{v j}(\hbar \omega)\right] \delta\left(\hbar \omega-E_{e i h j}\right)
$$

Where $\delta(x)$ represents the Heaviside step function, $f_{c i}$ and $f_{v i}$ are Fermi functions for the $j^{\text {th }}$ subband in the valence band and $i^{\text {th }}$ subband in the conduction band, respectively, $\left.|| i j\right|^{2}$ is the electron-hole wave function overlap $/\left\langle\psi_{e i}\left|\psi_{h i}\right|^{2}, E_{e i h j}\right.$ is the energy corresponding to the optical transition starting from the conduction to the valence bands and:

$$
G_{0}=\frac{e^{2} X_{C V}^{2} \mu^{3 / 2}}{n_{A Z} c \varepsilon m_{0}^{2} \eta^{2} \omega L_{e f f}}
$$

Where $\mu=\left(m_{e}^{-1}+m_{h}^{-1}\right)$ is the electron-hole reduced effective mass, $c, \omega$ and $\varepsilon$ are respectively the speed of light, the pulsation of light and the effective dielectric constant of the material; $n_{A Z} L_{\text {eff }}$ are the refractive index and the effective width of the active zone, respectively, and $X_{C V}^{2}=h^{2} E_{p} / 3 m_{0} E_{e i h j}^{2}$ where $E_{p}=\left(2 / m_{0}\right) P^{2}$ with $P$ is the Kane matrix element.

In concerned cases, the optical gain has to be convoluted by some broadening function, so as to account for the limited
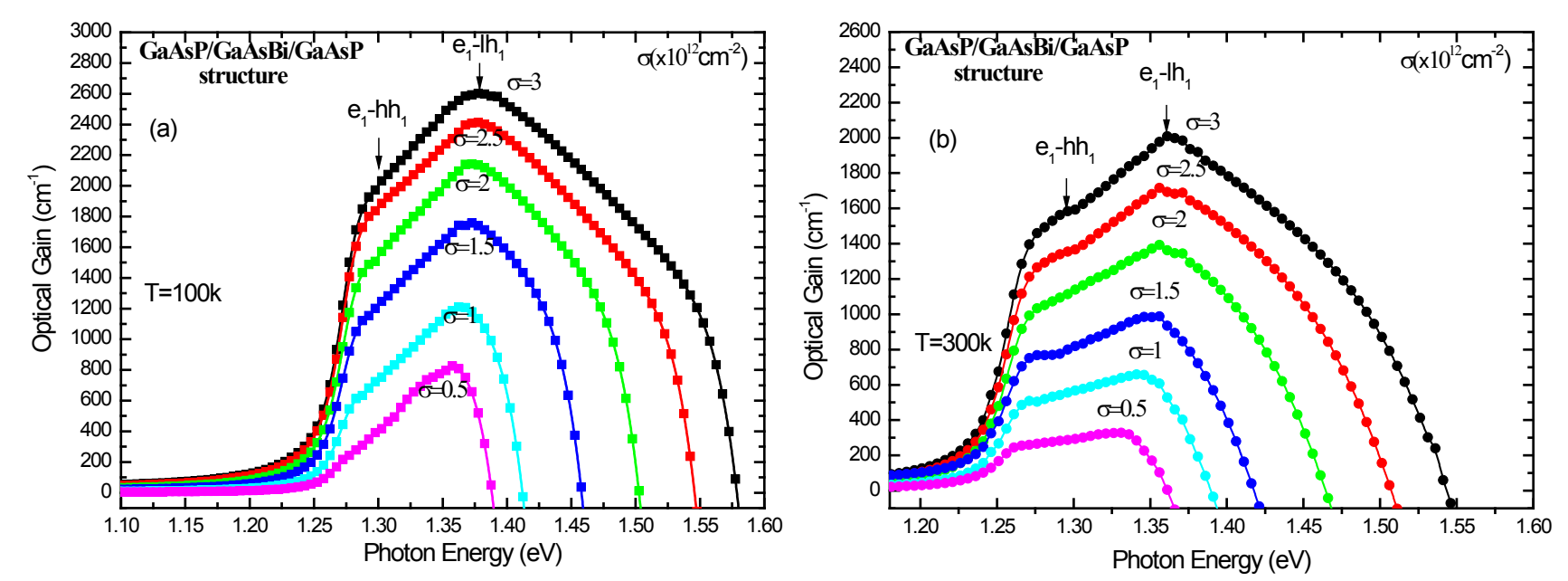

Figure 4: Gain spectra (TE mode) versus photon energy in a single GaAsBi/GaAsP QW structure for different carrier injection densities varying from $\sigma=0.5 \times 10^{12} \mathrm{~cm}^{-2}$ to $\sigma=3 \times 10^{12} \mathrm{~cm}^{-2}$. Calculations are made at $T=100 \mathrm{~K}(\mathrm{a})$ and $\mathrm{T}=300 \mathrm{k}(\mathrm{b})$, respectively.

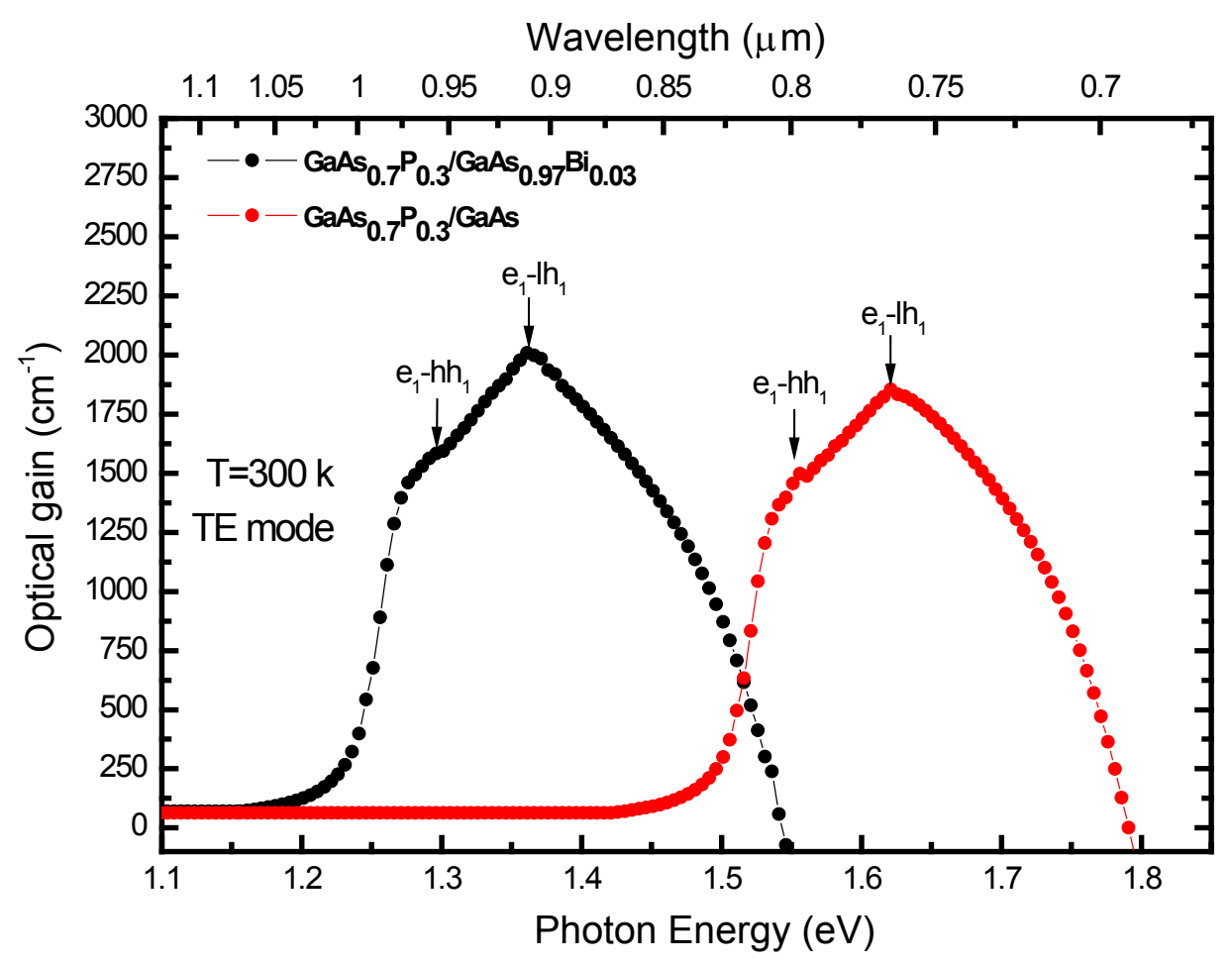

Figure 5: RT optical gain spectrum versus photon energy at carrier injection density $N_{2 D}=3 \times 10^{12} \mathrm{~cm}^{-2}$ Calculated for dilute-Bi in GaAsP/ GaAsBi/GaAsP and Bi-free GaAsP/GaAs/GaAsP structures. 
lifetime of excitons. Thus, we shall use a Lorentzian shape for this broadening, with a half width at half maximum denoted by $\Gamma$. Also, we assume a $\Gamma$ value equal to $26 \mathrm{meV}$ leading to an intraband relaxation time $\tau_{\text {int }}$ which is worth $100 \mathrm{fs}$ [34].

The optical gain is written as follow:

$$
\tilde{G}(\hbar \omega)=\int_{-\infty}^{+\infty} G(\hbar \omega-E) \frac{\left(\Gamma_{0} / 2\right)}{\pi\left(E^{2}+\left(\Gamma_{0}^{2} / 4\right)\right)} d E
$$

where $\tilde{G}(\hbar \omega)$ is given by equation (3).

The optical gain per GaAs$s_{0.7} \mathrm{P}_{0.3} / \mathrm{GaAs}_{0.7} \mathrm{P}_{0.3} / \mathrm{GaAs}_{0.97} \mathrm{Bi}_{0.03} /$ $\mathrm{GaAs}_{0.7} \mathrm{P}_{0.3}$ period was calculated for different carrier injection densities $\sigma$ in the active region varying from $0.5 \times 10^{12}$ to $3 \times 10^{12} \mathrm{~cm}^{-2}$ Obtained results are plotted in Figure 4 at low $(T=100 \mathrm{~K})$ and $\mathrm{RT}(T=300 \mathrm{~K})$. At low temperature on Figure $4 a$, we guess that, it is easy to get gain because the difference between electron and hole quasi-Fermi energies $\left(E_{f c}-E_{f v}\right)$ reaches the $\left(\mathrm{e}_{1}-\mathrm{hh}_{1}\right)$ transition energy starting from the carrier injection of $\sigma=0.5 \times 10^{12} \mathrm{~cm}^{-2}$. On the other hand, at RT Figure $4 \mathrm{~b}$, it is compulsory to have an injection rate of at least $\sigma=1 \times 10^{12} \mathrm{~cm}^{-2}$ to allow to carriers to fulfill stimulated emission conditions. Whatever the temperature, the maximum gain $G_{\max }$ due to the first energy level corresponding to the transition $\left(\mathrm{e}_{1}-\mathrm{h} \mathrm{h}_{1}\right)$ increases quasi-linearly with the injected carrier density. This gain peak is dominated at low temperature by the $\left(\mathrm{e}_{1}-\mathrm{Ih}_{1}\right)$ transition only for lower injection rates. Furthermore, At $\sigma=$ $1.5 \times 10^{12} \mathrm{~cm}^{-2}$, the gain from the second quantized level transition $\left(e_{1}-h h_{1}\right)$ involving light hole carriers becomes significant. Moreover, The transparency carrier density is equal to $0.25 \times 10^{12} \mathrm{~cm}^{-2}$ at RT. For a typical injected carrier density of $2 \times 10^{12} \mathrm{~cm}^{-2}$, a gain value of about 1500 $\mathrm{cm}^{-1}$ is obtained, which is sensibly comparable to values obtained on $\mathrm{GaSbBi} / \mathrm{GaSb}$ structure tailored for emission at $2 \mu \mathrm{m}(\mathrm{E}=0.62 \mathrm{eV})$ on GaSb substrate [35]. This steep gain value could be attributed to the $2 \mathrm{D}$ density of states which is proportional to the effective masses and inversely proportional to the confinement energy of electron and hole as well as to the quasi-Fermi levels [36]. From the viewpoint of applications, the optical quality of dilute bismide in lasers is a very valuable issue. Indeed, for $\mathrm{GaAsBi}$ and InPBi, this quality dwindles very significantly due to incorporation of $\mathrm{Bi}$ atoms [37]. The Figure 5 shows the optical gain spectra of the two laser structures, with dilute-Bi and $\mathrm{Bi}$-free. For two-dimensional system, carrier densities $\mathrm{N}_{2 \mathrm{D}}$ is rises up to $3 \times 10^{12} \mathrm{~cm}^{-2}$. In the case of the dilute-Bi structure, the conduction subbands $e_{1}$ and $e_{2}$ are clearly splitted, consequently; for the used carrier densities in our calculations, only $e_{1}$ and $h h_{1}$ levels are occupied. Thus, the peak in the gain curve corresponds to $\left(e_{1}-h h_{1}\right)$ transition. Also, we note an improvement of optical gain values and a red shift of the gain spectrum to higher wavelengths in the based GaAsBi QW laser structure [38]. The latter could be explained by the large bowing factor of the GaAsBi energy band gap. For dilute-Bi structure, a gain value at around of $2000 \mathrm{~cm}^{-1}$ is attained with a typical injected carrier density of $3 \times 10^{12} \mathrm{~cm}^{-2}$. This result is comparable to those obtained on the GaSbBi/GaSb structure adapted for emission at 2 $\mu \mathrm{m}$ on GaSb substrate but higher than those obtained for Bi-free structure as illustrated in Figure 5 . These previous high gain values could be attributed in part to the electron mass enhancement as well as the increase in density of states in dilute-bismide compounds. For the Bi-free structure, when the carrier density is $3 \times 10^{12} \mathrm{~cm}^{-2}$, the gain peak corresponding to $\left(e_{1}-/ h_{1}\right)$ transition occurs at about 1.4259 $\mathrm{eV}$ and is larger than that of dilute-Bi which is worth 1.38 eV for the same carrier density. Moreover, for the Bi-free structure, the transition between the occupied $e_{1}$ and the unoccupied $e_{2}$ is unlikely. Thus, the difference in shape between the corresponding black and red curves could be interpreted by band filling and the resulting shift of the

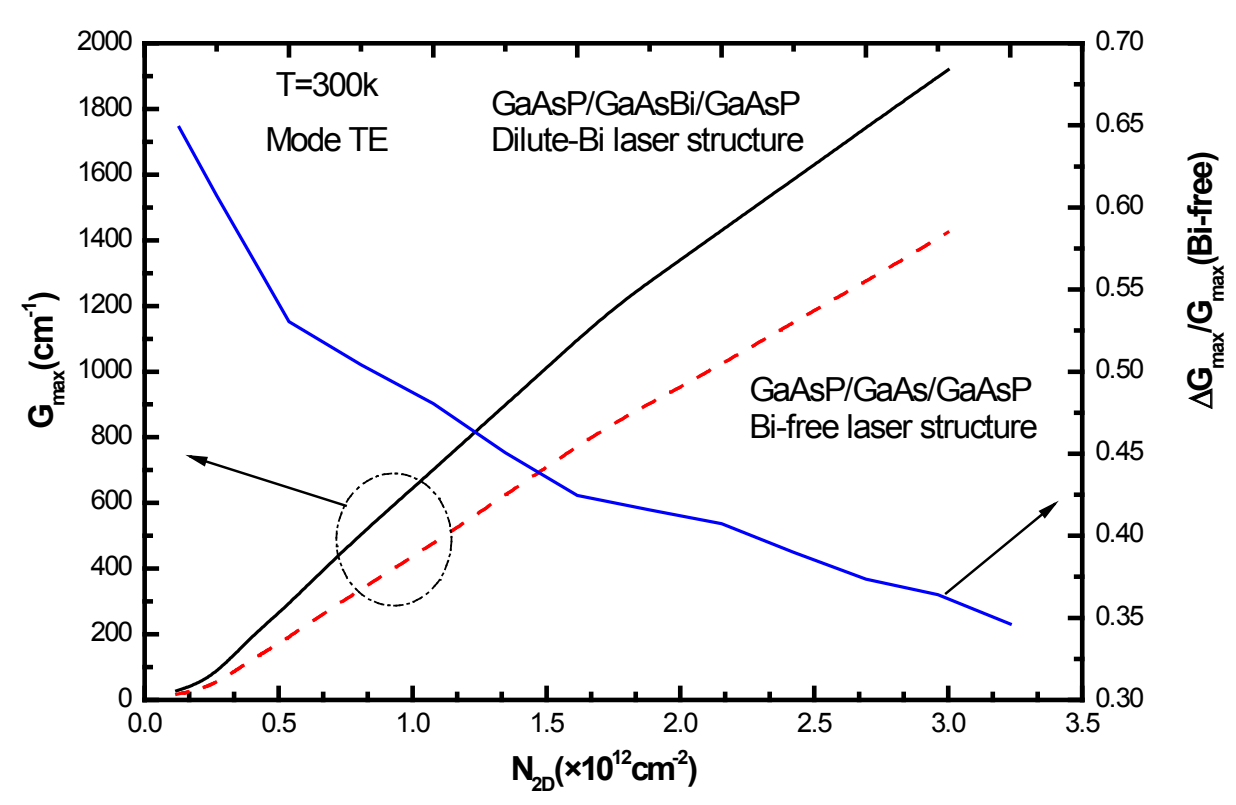

Figure 6: Left axis RT gain vs injected carrier density for the dilute-Bi (solid line) and the Bi-free (dashed line) structures. Right axis: Relative difference between the maximum gains for the two structures $\Delta G_{\max } / G_{\max }$ as a function of carrier densities. 


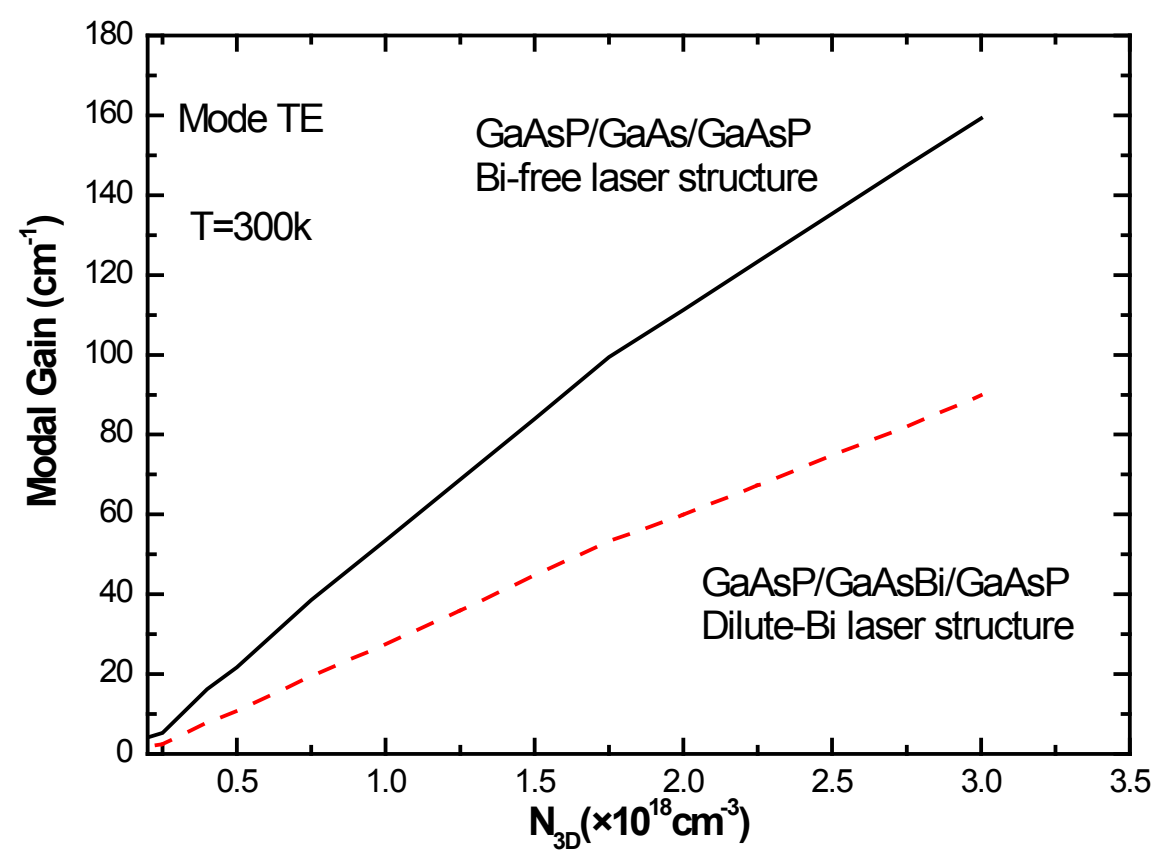

Figure 7: RT modal gain $\mathrm{G}_{\text {mod }}$ versus injected carrier concentration $\mathrm{N}_{3 \mathrm{D}}\left(\mathrm{cm}^{-3}\right)$ for the dilute-Bi (solid line) and the Bi-free (dashed line) laser structures composed of five $\mathrm{GaAs}_{0.7} \mathrm{P}_{0.3} / \mathrm{GaAs}_{0.97} \mathrm{Bi}_{0.03}$ periods.

quasi-Fermi energy separation. Due to the variation of the effective mass at the level of the valence band in $\mathrm{GaAsBi}$ with the incorporation of bismide as well as the densities of states, these results leading to a higher transparency current similar to InGaAs-based laser structures $[39,40]$.

In addition the optical gain of GaAsBi-based laser structure is comparable with the optical gain values in InGaAs-based devices [36]. Calculated maximum gain as a function of carrier density is illustrated in Figure 6 for both structures at RT. This shows the superiority of the dilute-Bi structure in comparison to the Bi-free one, whatever these have the same carrier density. In the case of a carrier density equal to $3 \times 10^{12} \mathrm{~cm}^{-2}$, the incorporation of bismide in $\mathrm{GaAs}$ well ( $\mathrm{GaAsBi}$ ) induces an enhancement in the $G_{\max }$ value. The transparency carrier density is $5.5 \times$ $10^{18} \mathrm{~cm}^{-3}$ for the dilute $\mathrm{Bi}$ and $5.42 \times 10^{18} \mathrm{~cm}^{-3}$ for the $\mathrm{Bi}$ free structures. These latter values are obtained using the relation $\mathrm{N}_{3 \mathrm{D}}=\mathrm{N}_{2 \mathrm{D}} / L_{\text {eff }}$, where $L_{\text {eff }}$ is the effective width of the structure equal to $222 \AA$ and $225 \AA$ for dilute- $\mathrm{Bi}$ and $\mathrm{Bi}$-free structures, respectively.

It is well acknowledged that the suitable part of the optical gain (modal gain) has to recompense the total optical losses $(\alpha)$, of the laser diode for this reason we,

The most interesting result was obtained for dilute-Bi structure with a $\Gamma_{p}$ value per period of $1.66 \%$ while $\Gamma_{p}$ is equal to $1.26 \%$ for the Bi-free structure [41]. The Figure 7 illustrates the variation of modal gain versus carrier concentration $\mathrm{N}_{3 \mathrm{D}}$ for dilute-Bi and Bi-free structures. Unsurprisingly, the maximum modal gain value is accomplished GaAsP/GaAsBi/GaAsP laser design. For a typical carrier concentration $\mathrm{N}_{3 \mathrm{D}}=1 \times 10^{18} \mathrm{~m}^{-3}\left(\sigma=1 \times 10^{12} \mathrm{~m}^{-3}\right)$, the modal gain at $\mathrm{T}=300 \mathrm{~K}$ exceeds $54 \mathrm{~cm}^{-1}$, whereas it reaches only
$28 \mathrm{~cm}^{-1}$ for the Bi-free structure. Thus, this result confirms the valued effect of the dilute-bismide incorporation in the active region of laser structure and corroborates the possibility to obtain, at RT, a laser structure operating with reasonable optical losses. Starting from the relation $G_{\text {mad }}$ $=G_{p} N_{p} G_{\text {max }}$, we have performed the calculation the modal gain $G_{\text {mad }}$ where $G_{\text {max }}$ is the peak gain value found out from the optical gain curves (Figure 5), Np is the quantum wells number taken equal to 5 and $G_{p}$ is the TE polarization optical confinement factor. Moreover, it was computed using the refractive indices $n_{\text {op }}$ extracted from Table 1, following the procedure assumed in Ref. [24]. The carried out calculations at $300 \mathrm{~K}$ indicated that the incorporation of bismide reduces the radiative efficiency of the structure (Figure $8 \mathrm{a})$. By assuming uniform charge injection across the laser active region, we can use the simplified expression of spontaneous emission rate given by [42].

$r_{s p}(E)=\frac{16 e^{2} n_{r} E}{m_{0}^{2} \hbar^{2} C^{3}} \sum_{i j}\left\langle\left|P_{c_{i}-v_{j}}\right|^{2}\right\rangle \rho_{\text {red }} f\left(E_{C_{i}}-F_{C}\right)\left[1-f\left(E_{v_{f}}-F_{v}\right)\right]$

Where $c$ is the speed of light, $n_{r}$ are the refractive index, $m_{0}$ is the free-electron mass, $\left\langle\left|P_{c_{i}-v_{j}}\right|^{2}\right\rangle$ is the squared momentum matrix element between electron and hole in each sub-band averaged over directions and polarizations of photons and spins of electrons and holes, $\rho_{\text {red }}$ is the reduced density of state of electrons and holes in the $x y$ plan without spin degeneracy, $F_{c, v}$ is the quasi-Fermi level of conduction and valence band and $f(E)$ the Fermi-Dirac distribution function $\rho_{\text {red }}$ is written as $m_{\text {red }} / 2 \pi \hbar^{2}$ through the effective mass approximation, where $m_{\text {red }}$ is reduced mass of electrons and holes in the xy plane. $\left\langle\left|P_{c_{i}-v_{j}}\right|^{2}\right\rangle$ is estimated by the $\mathrm{k} \cdot \mathrm{p}$ theory to be : 

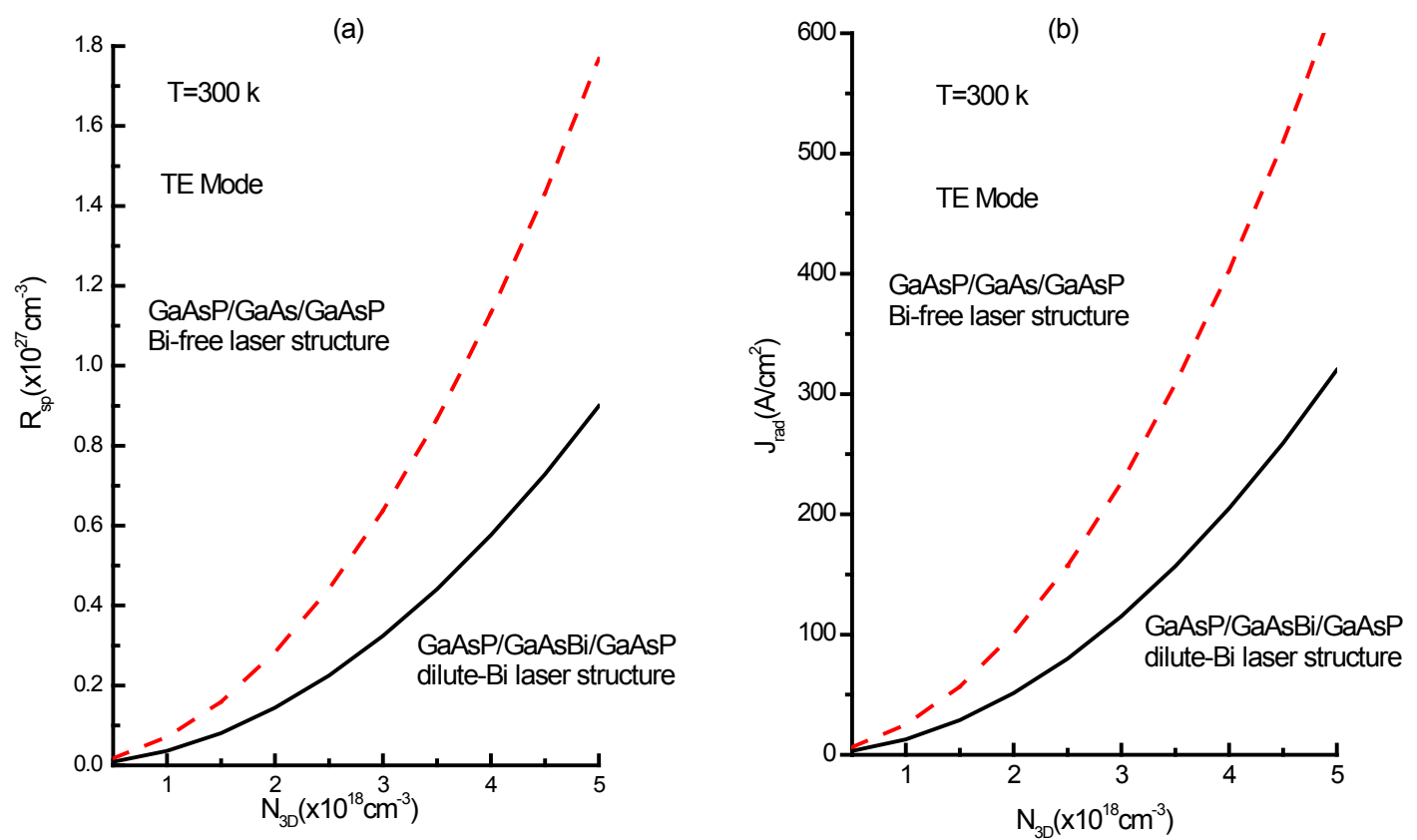

Figure 8: (a) Spontaneous emission rate $\mathrm{R}_{\mathrm{sp}}$ and (b) Radiative current density $\mathrm{J}_{\text {rad }}$ versus injected carrier concentration $\mathrm{N}_{3 \mathrm{D}}\left(\mathrm{cm}^{-3}\right)$ calculated at $\mathrm{T}=300 \mathrm{~K}$ for dilute-Bi (solid line) and $\mathrm{Bi}$-free (dashed line) laser structures.

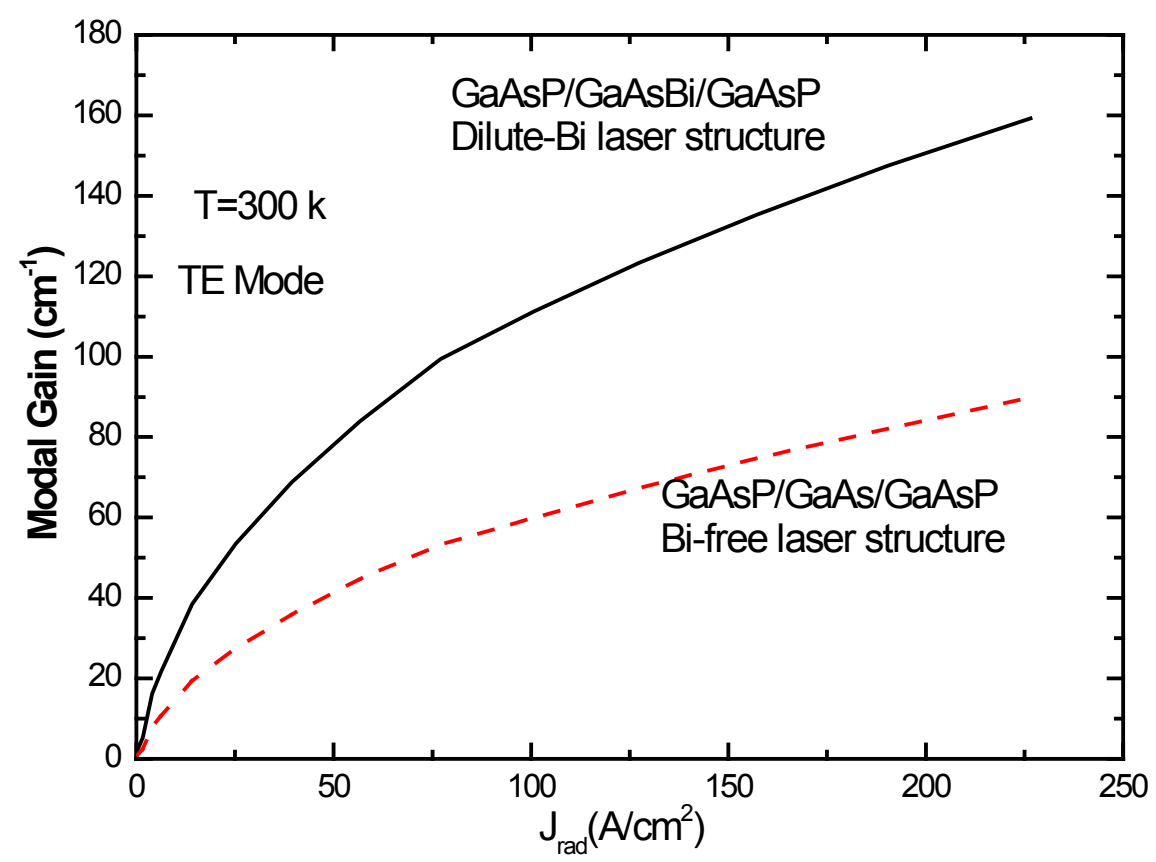

Figure 9: RT evolution of the TE polarized modal gain $G_{\text {mod }}$ versus radiative current density $\mathrm{J}_{\text {rad }}$ for the dilute-Bi (solid line) and the Bi-free (dashed line) laser structures.

$\left\langle\left|P_{c_{i}-v_{j}}\right|^{2}\right\rangle=\left\langle\left|P_{e_{i}-h h_{j}}\right|^{2}\right\rangle=\left\langle\left|P_{e-l h_{j}}\right|^{2}\right\rangle=\frac{\left(m_{0} / m_{C}-1\right)\left(m_{0} E_{g} / 12\right)\left(E_{g}+\Delta\right)}{\left(E_{g}+2 \Delta / 3\right)} \eta_{c i-v j}^{2}$

Where $m c$ is the electron effective mass, $E g$ the band gap energy , $\Delta$ the spin-orbit splitting and $\eta c-v$ the overlap integral of the envelope function of the electrons and holes, respectively. A low bound on the threshold current density may be obtained by calculating the radiative recombination rate per unit volume given by [42].

$$
R_{s p}\left(N_{3 D}, T\right)=\int r_{s p}(E) d E
$$

Where $\mathrm{E}$ is the photon energy. The performed calculation results at $300 \mathrm{~K}$ leads to believe that the addition of bismide degrades the radiative efficiency of the structures (Figure 8a). By taking into account uniform charge injection across the laser active region, we can use the simplified expression: 


$$
R_{s p}\left(N_{3 D}, T\right)=B(T) N_{3 D}^{2}
$$

The radiative recombination coefficient $B$ at $R T$ equal to $3.6 \times 10^{-11}$ and $7.076 \times 10^{-11} \mathrm{~cm}^{3} \mathrm{~s}^{-1}$, respectively, for dilute-Bi and Bi-free laser structures, in agreement with standard values of GaAs laser structures $[43,44]$. Furthermore, the radiative current density ${ }_{\mathrm{rad}}$ could be calculated by integrating over the spontaneous emission spectrum at each value of $\mathrm{N}_{30}$ [45], it comes:

$$
J_{\text {rad }}=e L_{z} \int r_{s p}(E) d E
$$

where e is the electron charge and $L_{z}=N_{p} L_{\text {eff }}$ is the total active region. For typical injected carrier density $N_{30}=$ $2.5 \times 10^{18} \mathrm{~cm}^{-3}$, typical for laser diode operation at RT. The $J_{r a d}$ values are always less than $100 \mathrm{~A} / \mathrm{cm}^{2}$ for the dilute-Bi structure, confirming the possibility to such laser diode to operate at RT. These relevant results are illustrated in Figure $8 \mathrm{~b}$, where the modal gain $\mathrm{G}_{\bmod }$ versus the radiative current density $J_{\text {rad }}$ at $300 \mathrm{~K}$ for the two LDs under consideration at RT indicate in Figure 9. The result reported in Figure 9 demonstrates that modal gain is better for the dilute-Bi structure, about two times higher than the Bi-free structure all over the $J_{\text {rad }}$ values. Keeping in view reasonable total optical losses at around $10-15 \mathrm{~cm}^{-1}$ for a low-defect laser structure operating at RT [46], the $J_{\text {rad }}$ value is deduced for the dilute-Bi structure two times lower than the Bi-free one. Basing upon these theoretical investigations, it can be guessed that $\mathrm{GaAsBi} / \mathrm{GaAs}$ quantum well lasers structures are a promising new class of near-infrared devices where, by use of the unusual band structure properties of $\mathrm{GaAsBi}$ alloys, it is possible to eliminate the dominant energy-consuming Auger recombination and inter-valence band absorption loss mechanisms, which have a great impact on the device performance [47]. We emphasize that the inhibition of these loss mechanisms let us hope GaAsBi to be the potential candidate for the development of next-generation semiconductor lasers. The precise estimation of the Auger coefficient in the diluted-Bi laser structures will be the subject of future studies.

\section{Conclusion}

A new dilute-Bi type-I GaAs ${ }_{0.7} \mathrm{P}_{0.3} / \mathrm{GaAs}_{0.97} \mathrm{Bi}_{0.03}$ laser structure has been proposed and optimized for emission at 0.960 $\mu \mathrm{m}$ and at RT in order to show an appropriate overlap wave functions value equal to $70 \%$. The theoretical performances of such laser structure are compared to a similar conventional Bi-free structure for laser operation at RT. The results obtained on the calculation of the optical gain for the two laser structures show the superiority of the bismuth system due to the increased effective mass of the edge of the valance band in the GaAsBi layer and the improved optical density of states in dilute-Bi compounds. As a consequence, the dilute-Bi laser structure presents high optical material gain around $1000 \mathrm{~cm}^{-}$ 1 , modal gain value is equal to $80 \mathrm{~cm}^{-1}$ and radiative current density below $100 \mathrm{~A} / \mathrm{cm}^{2}$ at $300 \mathrm{~K}$ for typical injected carrier concentration $N_{3 D}=1.5 \times 10^{13} \mathrm{~cm}^{-3}$. These results approve that the GaAsP/GaAsBi structure are of an excessive attention for the realization of laser devices operating in the near-infrared region at $\mathrm{RT}$.

\section{References}

1. Takamoto T, Kaneiwa M, Imaizumi M, et al. (2005) InGaP/GaAsbased multijunction solar cells. Prog Photovoltaics 13: 495.

2. Yamaguchi M, Nishimura K, Sasaki T, et al. (2008) Investigation of LPE grown dilute nitride InGaAs(Sb)N layers for photovoltaic applications. Sol Energy 82: 173.

3. Dimroth F (2006) High-efficiency solar cells from III-V compound semiconductors. Phys Status Solidi C 3: 373.

4. Bertulis K, Krotkus A, Aleksejenko G, et al. (2006) GaBiAs: A material for optoelectronic terahertz devices Appl Phys Lett 88: 201112.

5. Tominaga $Y$, Kinoshita $Y$, Oe K, et al. (2008) Structural investigation of $\mathrm{GaAs}_{1-\mathrm{x}} \mathrm{Bi}_{\mathrm{x}} / \mathrm{GaAs}$ multiquantum wells. Appl Phys Lett 93: 131915.

6. Fluegel B, Francoeur S, Mascarenhas A, et al. (2006) Giant spin-orbit bowing in $\mathrm{GaA}_{\mathrm{s1-}-\mathrm{Bi}} \mathrm{Bi}_{\mathrm{x}}$. Phys Rev Lett 97: 067205.

7. Lu XF, Beaton DA, Lewis RB, et al. (2009) Composition dependence of photoluminescence of $\mathrm{GaAs}_{1-\mathrm{x}} \mathrm{Bi}_{\mathrm{x}}$ alloys. Appl Phys Lett 95: 041903.

8. Mohmad AR, Bastiman F, Ng JS, et al. (2011) The effect of Bi composition to the optical quality of $\mathrm{GaAs}_{1-\mathrm{x}} \mathrm{Bi}_{\mathrm{x}}$. Appl Phys Lett 98: 122107.

9. Yeh JY, Mawst L, Khandekar AA, et al. (2006) Characteristics of InGaAsN-GaAsSb type-II "W" quantum wells. J Cryst Growth 287: 615-619.

10. Chen B (2017) Optical gain analysis of GaAs-based InGaAs/ GaAsSbBi type-II quantum wells lasers Optics Express 25: 2518325192.

11. Alberi K, Wu J, Walukiewicz W, et al. (2007) Valence-band anticrossing in mismatched III-V semiconductor alloys. Phys Rev B 75: 045203.

12. Lewis $R$, Beaton $D$, Lu X, et al. (2009) $\mathrm{GaAs}_{1-\mathrm{x}} \mathrm{Bi}_{\mathrm{x}}$ light emitting diodes. J Cryst Growth 311: 1872.

13. Hossain N, Marko IP, Jin SR, et al. (2012) Recombination mechanisms and band alignment of $\mathrm{GaAs}_{1-\mathrm{x}} \mathrm{Bi}_{\mathrm{x}} / \mathrm{GaAs}$ light emitting diodes. Appl Phys Lett 100: 51105.

14. Tominaga $Y$, Oe K, Yoshimoto $M$ (2010) Low temperature dependence of oscillation wavelength in $\mathrm{GaAs}_{1-\mathrm{x}} \mathrm{Bi}_{\mathrm{x}}$ laser by photo-pumping. Appl Phys Express 3: 62201.

15. Ludewig P, Knaub N, Hossain N, et al. (2013) Electrical injection $\mathrm{Ga}(\mathrm{AsBi}) /(\mathrm{AlGa}) \mathrm{As}$ single quantum well laser. Appl Phys Lett 102: 242115.

16. Lu X, Beaton DA, Lewis RB, et al. (2008) Effect of molecular beam epitaxy growth conditions on the $\mathrm{Bi}$ content of $\mathrm{GaAs}_{1-\mathrm{x}} \mathrm{Bi}_{x} \mathrm{Appl}$ Phys Lett 92: 192110.

17. Pacebutas V, Bertulis K, Aleksejenko G, et al. (2009) Molecular-beam-epitaxy grown GaBiAs for terahertz optoelectronic applications. J Mater Sci Mater Electron 20: 363-366.

18. Imhof S, Thranhardt A, Chernikov A, et al. (2010) Clustering effects in Ga(AsBi). Appl Phys Lett 96: 131115.

19. Grein CH, John S (1989) Temperature dependence of the Urbach optical absorption edge: A theory of multiple phonon absorption and emission sidebands. Phys Rev B 39: 1140. 
20. Hjalmarson $H$, Vogl P, Wolford DJ, et al. (1980) Theory of substitutional deep traps in covalent semiconductors. Phys Rev Lett 44: 810

21. Shen J, Ren SY, Dow JD (1990) Relaxed-lattice model of isolated and paired isoelectronic traps in GaP. Phys Rev B Condens Matter 42: 9119-9126.

22. Francoeur S, Tixier S, Young E, et al. (2008) Bi isoelectronic impurities in GaAs. Phys Rev B 77: 085209.

23. Forghani K, Guan Y, Losurdo M, et al. (2014) $\mathrm{GaAs}_{1-\mathrm{y}-\mathrm{z}} \mathrm{P}_{\mathrm{y}} \mathrm{Bi}_{z^{\prime}}$ an alternative reduced band gap alloy system lattice-matched to GaAs. Appl Phys Lett 105: 111101.

24. Harbi N, Sfina N, Jbeli A, et al. (2015) Direct band gap $\ln _{x} G_{1-x} A s /$ Ge type II strained quantum wells for short-wave infrared $p-i-n$ photodetector. Optical Materials 46: 472-480.

25. Joullie A, Christol P (2003) GaSb-based mid-infrared 2-5 $\mu \mathrm{m}$ laser diodesDiodes laser à base GaSb pour moyen infrarouge (2-5 $\mu \mathrm{m})$. C R Phys 4: 621-637.

26. Ferhat M, Zaoui A (2006) Structural and electronic properties of III-V bismuth compounds. Phys Rev B 73: 115107.

27. Alberi K, Walukiewicz W, Yu KM, et al. (2007) Haas measurement of electron effective mass in $\mathrm{GaAs}_{1-\mathrm{x}} \mathrm{Bi}_{\mathrm{x}}$. Appl Phys Lett 91: 051909.

28. Usman M, Broderick CA, Batool Z, et al. (2013) Impact of alloy disorder on the band structure of compressively strained $\mathrm{GaBi}_{\mathrm{x}}$ $\mathrm{As}_{1-x^{*}}$. Phys Rev B 87: 115104.

29. Kopaczek J, Kudrawiec R, Polak MP, et al. (2014) Contactless electroreflectance and theoretical studies of band gap and spin-orbit splitting in $\operatorname{In} \mathrm{P}_{1-x} \mathrm{Bi}_{x}$ dilute bismide with $\mathrm{x} \leq 0.034$. Appl Phys Lett 105: 222104.

30. Kim H, ForghaniK, Guan Y, et al. (2015) Strain-compensated $\mathrm{GaAs}_{1-\mathrm{y}} \mathrm{P}_{\mathrm{y}} / \mathrm{GaAs}_{1-z} \mathrm{Bi}_{z} / \mathrm{GaAs}_{1-\mathrm{y}} \mathrm{P}_{\mathrm{y}}$ quantum wells for laser applications. Semicond Sci Technol 30: 094011.

31. Ahn D, Chuang SL (1990) Optical gain and gain suppression of quantum-well lasers with valence band mixing. IEEE J Quantum Electron 26: 13-24.

32. Tansu N, Mawst $\amalg$ (2005) Current injection efficiency of InGaAsN quantum-well lasers. J Appl Phy 97: 054502.

33. Robert C, Perrin M, Cornet C, et al. (2012) Atomistic calculations of $\mathrm{Ga}(\mathrm{NAsP}) / \mathrm{GaP}(\mathrm{N})$ quantum wells on silicon substrate: Band structure and optical gain. Appl Phys Lett 100: 111901.
34. Gladysiewicz M, Kudrawiec R, Miloszewski JM, et al. (2013) Band structure and the optical gain of GalnNAs/GaAs quantum wells modeled within 10-band and 8-band kp model. J Appl Phys 113: 063514.

35. A Ben Ahmed, H Saidi, et al. (2015) Band structure, optical transition, and optical gain of Type-II InAs(N)/GaSb quantum wells laser diodes modeled within 16-band and 14-band kp model. IEEE J Quantum Electron 51: 2500108.

36. Igor P Marko, Christopher A Broderick, Shirong Jin, et al. (2016) Optical gain in $\mathrm{GaAsBi} / \mathrm{GaAs}$ quantum well diode lasers Sci Rep 6: 28863.

37. Tourniee E, Baranov AN (2012) Chapter 5 - Mid-infrared semiconductor lasers: A review. Semiconductors and Semimetals 86: 183-226.

38. Igor P Marko, Stephen J Sweeney (2017) Progress Toward III-V Bismide Alloys for Near- and Midinfrared Laser Diodes. IEEE J Sel Top Quant Electron 23: 1501512.

39. Tsvid G, Kirch J, Mawst LJ, et al. ( 2008) Spontaneous radiative efficiency and gain characteristics of strained-layer InGaAs-GaAs quantum-well lasers. IEEE J Quantum Electron 44: 732.

40. Chuang SL (1995) Physics of optoelectronic devices, Wiley Series in Pure and Applied Optics, New York.

41. Lash G, Stern F (1964) Spontaneous and stimulated recombination radiation in semiconductors. Phys Rev A 133: 553-563.

42. Bebb HR, Wiliams EW (1972) Semiconductors and Semimetals. In: Willardson RK Beer AC, Academic, New York.

43. Christol C, Bigenwald P, Joullie A, et al. (1999) Improvement of Sb-based multiquantum well lasers by Coulomb enhancement. IEE Proc Optoelectron 146: 3-8.

44. Christopher A Broderick, Patrick E Harnedy, Eoin PO'Reilly (2015) Theory of the Electronic and Optical Properties of Dilute Bismide Quantum Well Lasers. IEEE Quntum electronics 21.

45. Bishop PJ, Daniels ME, Ridley BK, et al. (1992) Radiative recombination in $\mathrm{GaAs} / \mathrm{Al}_{\mathrm{x}} \mathrm{Ga}_{1-\mathrm{x}}$ As quantum wells. Phys Rev B 45: 6686.

46. Fluegel B, Francoeur S, Mascarenhas A, et al. (2006) Giant Spin-Orbit Bowing in $\mathrm{GaAs}_{1-\mathrm{x}} \mathrm{Bi}_{\mathrm{x}}$. Phys Rev Lett 97: 067205.

47. Imhof S, Buckers C, Thranhardt A, et al. (2008) Microscopic theory of the optical properties of $\mathrm{Ga}(\mathrm{AsBi}) / \mathrm{GaAs}$ quantum wells. Semicond Sci Technol 23: 125009.

\section{DOI: $10.36959 / 665 / 321$}

Copyright: (c) 2019 Sfina N, et al. This is an open-access article distributed under the terms of the Creative Commons Attribution License, which permits unrestricted use, distribution, and reproduction in any medium, provided the original author and source are credited. 